\title{
CLUSTER CONTROL OF COMPLEX CYBER-PHYSICAL SYSTEMS
}

\author{
Denis Uzhva \\ Laboratory "Control of Complex Systems" \\ Institute for Problems \\ in Mechanical Engineering \\ Faculty of Mathematics and Mechanics \\ Science Educational Center \\ "Mathematical Robotics and Artificial Intelligence" \\ Saint Petersburg State University \\ St. Petersburg, Russia \\ denis.uzhva@yahoo.com
}

\author{
Oleg Granichin \\ Laboratory "Control of Complex Systems" \\ Institute for Problems \\ in Mechanical Engineering \\ Faculty of Mathematics and Mechanics \\ Science Educational Center \\ "Mathematical Robotics and Artificial Intelligence" \\ Saint Petersburg State University \\ St. Petersburg, Russia \\ o.granichin@spbu.ru
}

Article history:

Received 05.10.2021, Accepted 25.11.2021

\begin{abstract}
To our minds, the real world appears as a composition of different interacting entitites, which demonstrate complex behavior. In the current paper, we primarly aim to study such networked systems by developing corresponding approaches to modeling them, given a class of tasks. We derive it from the primary concept of information and a system, with corresponding dynamics emerging from interactions between system components. As we progress through the study, we discover three possible levels of certain synchronous pattern composition in complex systems: microscopic (the level of elementary components), mesoscopic (the level of clusters), and macroscopic (the level of the whole system). Above all, we focus on the clusterization phenomenon, which allows to reduce system complexity by regarding only a small number of stable manifolds, corresponding to cluster synchronization of system component states-as opposed to regarding the system as a whole or each elementary component separately. Eventually, we demonstrate how an optimization problem for cluster control synthesis can be formulated for a simple discrete linear system with clusterization.
\end{abstract}

\section{Key words}

control of complex systems, multiagent technologies, discrete systems

\section{Introduction}

Since the ancient times, humanity was always deeply curious about reasons and causes of things. At the most basic level, some objects appear to our minds as identical, while other ones can be highlighted from the rest by some distinctive features. In fact, this ability to generalize the perceived environment-but not in all its manifestations, just in certain patterns-originates mathematics and cybernetics. These two areas of knowledge can in fact represent two interconnected ways of understanding information and (complex) systems.

Mathematics plays the key role in the objectives, where a human needs to replicate his ideas in an accessible (for the others) and compact form, and, moreover, reproduce these ideas exactly the same as they appear to the person they originate from. For example, ancient Babylonian mathematics was exploited in engineering and architectural tasks, where a resulting product should have been precisely geometrically implemented from human mind. In this time period, geometry and arithmetic were proposed to help generalize and manipulate mental representations of real objects, for the previously unknown ones to be created. Indeed, instead of trying to enumerate the continuum of internal and boundary points of a triangle, it is sufficient to specify the locations of the three vertices and grasp the concept of a straight line segment between two points. In other words, mathematics practically allows to compress signals and data:

1. Signals, perceived from the environment (by finding common patterns in nature).

2. Data, produced in a mind by combining abstract generalized concepts (e.g. number, figure) according to some meaningful rules (for example, arithmetic). 
This peculiarity of the mathematical approach brings us to the first way of understanding information-it is matter, which appears to our minds identical on local level (in a certain sense of locality, probably in some transformed perception standard, where we agree to consider certain things identical), and which looks distinct on the corresponding global level (according to the same sense of locality). As an example, consider a table, which we highlight from other objects in a room. On its local level (in the volume of space this table occupy), it is composed of roughly the same molecules, so that our mind notices this property and generalizes the whole bunch of molecules into a single object, aiming to avoid storing more than enough to sufficiently describe the table. At the same time, on the global level of the whole room, a table can be clearly distinguished from other room furniture-other systems.

Cybernetics is a much younger study than mathematics, which not only pursues the goal to develop theoretical tools for efficient organization of telecommunications and control of systems, but also aims to further provide deeper insight into information and complex systems as independent entities. Indeed, signal compression based on digital or analog encoding allows to extract meaningful components from the whole, so that the load on a corresponding telecommunication channel for data transfer would be minimal, and thus more data can be passed through the channel. At the same time, humans tend to compress data from perceived signals in the everyday life by their nature, so that more knowledge can be obtained and stored. Despite cybernetics and mathematics may seem very similar according to the example provided above, the main distinction between the studies is the presence and significance of time in the signals and systems. Here time arises from our ability to memorize things and thus distinguish the past from the present and future; for us, the world is subject to dynamics, to changes and transformations, which we reflect in our cybernetical models of complex systems. Moreover, any static mathematical example of finding common patterns can be regarded as a dynamic process, if we always take into account the human limitation of being able to be focused on only a few things at a time: we never gather data instantaneously, observation is always a time consuming process (i.e. it occurs always during some non-zero time). For example, comparison of different parts of environment while observing the table in the room consumes time to change our attention and gather enough data for the final concept of the table to emerge and become clear. To further motivate the need to highlight time and dynamical nature of things, consider irreversible chaotic systems, behavior of which is notably hard to predict in the future, if their initial state is provided inaccurately in the past or in the present. Such inaccuracy may arise in presence of unknown (stochastic) disturbances or noise, which we are unable to predict due to bio- or technological limitations. Thus, in the world of dynamic processes, information can be thought of as any awareness by the subject that something has changed, accompanied by awareness that the rest of the things stay unchanged. This definition is very similar to the static one, except now locality can also be regarded in space and time separately, which may become convenient in control of systems with complex unpredictable dynamics.

\section{Clusterization in Complex Systems}

First, we need to provide a definition of a complex system, for further motivation of complex systems study to become clear. Next, two ways of system modeling are described, with corresponding pros and cons of both mentioned. Finally, we discuss the possible peculiarities of complex systems dynamics (stability and clusterization), which emerge due to internal and external processes, relatively to the system.

\subsection{Understanding of Complex Systems}

As we study patterns and their evolution in time, we may find that some generalization and corresponding dynamic analysis problems may appear exceptionally challenging. For example, consider the task of forecasting, which requires us to predict the weather in future. As we dive into this problem, we may find that near future weather $W_{F}^{1}$ (about a few hours) is quite similar to the present one $W_{P R}$, so that future can be thought as of slightly transformed present:

$$
W_{F}^{1}=T\left(W_{P R}\right) \approx W_{P R},
$$

where $T$ is the corresponding transformation. However, as we progress through time by stacking these transformations $\left(T^{n}\left(W_{P R}\right)\right.$, where $T$ repeatedly applied to $W_{P R} n$ times, corresponding to a forecast for multiple weeks ahead), future

$$
W_{F}^{n}=T^{n}\left(W_{P R}\right)
$$

becomes less and less similar to present; moreover, in case present weather would be slightly different $\widetilde{W}_{P R}$ comparing to $W_{P R}$ (i.e. $\widetilde{W}_{P R} \approx W_{P R}$ ), then stacking the same transformations for $\widetilde{W}_{P R}$ would result in some future weather

$$
\widetilde{W}_{F}^{n}=T^{n}\left(\widetilde{W}_{P R}\right),
$$

which may differ significantly from $W_{F}^{n}$. In other words, $\widetilde{W}_{P R} \approx W_{P R}$ does not necessarily lead to $\widetilde{W}_{F}^{n} \approx W_{F}^{n}$. This is due to the nature of $T$, which is governed by the way various internal components and external disturbances (e.g. air molecules, the Sun) of the climate system interact between each other, forming a networked system. The amount of the components and interconnections between them leads to inability of deriving and computing precise model of $T$ using limited resources and obvious reasoning. Given the example above, we 
can now define a complex system as a composition of a large number of simple interacting elements, placed in some environment, with which they also perpetually interact. This leads to the resulting overall system behavior to be unpredictable (by classical approaches) on distant time horizon, given simple behavior of its componentsa special property, which we call emergence.

With the above being said, we can outline two main branches to dealing with complexity:

1. Quantitative complexity reduction: apply unjustified amount of resources.

2. Qualitative complexity reduction: apply unconventional study approaches.

Nowadays, quantitative approaches tend to become infeasible, due to the resource limitations mentioned above. Thus, we aim to develop advanced mathematical approaches to complex system modeling.

\subsection{Complex Systems Modeling}

As it was noted in Introduction, dynamic way of modeling allows for intuitively clear representation of systems, that evolve in time. Three approaches, which we will discuss further, are of the highest interest:

1. Discrete (automata) modeling [Ravazzi et al., 2021; Li et al., 2020; Silva, 2014].

2. Continuous dynamical systems modeling [Strogatz, 2000; Arnold and Silverman, 1987; Gazi and Fidan, 2007; Proskurnikov and Granichin, 2018; Granichin and Uzhva, 2020; Granichin et al., 2020; Fradkov, 2007].

3. Field theory modeling [Hu et al., 2019; Luo et al., 2021].

According to the first method, a complex system is composed of finite number of elementary autonomous units (further called agents), each of them having their individual state, which changes iteratively in time. A corresponding system model can be expressed in a difference equation; as an example, consider an autoregressive model:

$$
x_{i}[t]=c+\sum_{k=1}^{K} \theta_{k} x_{i}[t-k]+w_{i}[t],
$$

where $x_{i}[t]$ is the state of an agent $i$ at time $t, c$ is a constant, $\theta_{1}, \ldots, \theta_{p}$ are parameters, and $w_{i}[t]$ is a stochastic disturbance. At $p=1$, we obtain a so-called Markov process, which in fact is subject to lack of memory due to $x_{i}[t]$ being dependent only on its previous iteration $x_{i}[t-1]$. Model (1) can be augmented by adding nonlinearity and inter-connections between multiple neighbor agents. Moreover, probabilistic variations of the automata models based on the Markov chain formalism can be considered [Li et al., 2020]. We also include Poincaré and Lorenz maps [Strogatz, 2000], notwithstanding such models are conventionally associated with dynamical systems. Summing up, automata approach is convenient for describing stochastic discrete processes (therefore any discrete dynamical system can be viewed as an automata system), which is relevant in the context of large-scale systems with a numerous number of digital agents.

Despite the automata modeling utilizes iterative approach to describe system evolution and, accordingly, such models may have straightforward implementation, cyber-physical systems may often countinuously depend on time. A corresponding dynamical systems approach is thus favorable, as it allows to express a complex system (composed of $N$ agents) dynamics using ordinary differential equations:

$$
\dot{x}_{i}(t)=f_{i}\left(x_{i}(t), u_{i}(t), w_{i}(t)\right),
$$

where $x_{i}(t) \in \mathbb{R}^{n_{i}}$ is a so-called state vector of an agent $i \in \mathcal{N}=\{1, \ldots, N\}\left(n_{i}\right.$ is the number of variables required to describe the agent state), $u_{i}(t)$ is a local control action, and $w_{i}(t) \in \mathbb{R}^{m_{i}}$ is a stochastic disturbance. Moreover, this method of systems modeling is not prone to lack of memory phenomenon mentioned above, due to more freedom available over chosing time intervals $x_{i}(t)$ defined on. Continuous dynamical systems are also easy for precise analytical analysis,

Finally, field theory approach may be regarded as the most generalized one (perhaps derived from Equation (2) in the limit $N \rightarrow \infty$ ), since it is able to model a continuum of agents:

$$
\dot{x}(\lambda, t)=f(\lambda, x(\lambda, t), u(\lambda, t), w(\lambda, t)),
$$

where agents are now "enumerated" (or rather localized) by a point $\lambda$ in $\mathbb{R}$ or its equinumerous subset (i.e. also with the cardinality of the continuum).

Modern cybernetics is mainly focused on discrete and continuous dynamical system modeling, while field theory modeling appears too early to discuss about. Consequently, we further focus on dynamical systems with finite number agents, without losing a hope that the developed theory can be generalized into model (3).

\subsection{Clusterization}

In Equation (2), control input $u$ regulates the behavior of the system by setting the rules of the agent state change, depending on the states of neighbor agents and other environmental factors. Artificial complex systems are often required to change its global state in a controllable manner, so that the rules $u$ should lead to the specified global goal. Aiming to study control inputs $u$, we first should provide a clear description of possible goals, with corresponding models describing such goals rigorously. In [Fradkov, 2007], five types of goals are defined:

1. Stabilization (bringing the all agent states $x$ to their corresponding constant state vectors $x_{*}$ ):

$$
\lim _{x \rightarrow+\infty} x(t)=x_{*} .
$$


2. Tracking (bringing agent states to some function $x_{*}(t)$, perhaps different for each agent):

$$
\lim _{x \rightarrow+\infty}\left|x(t)-x_{*}(t)\right|=0 .
$$

3. Excitation of oscillations:

$$
\lim _{x \rightarrow+\infty} G(x(t))=G_{*}
$$

for some scalar function $G(x)$.

4. Synchronization (matching all agent states):

$$
\lim _{x \rightarrow+\infty}\left|x_{i}(t)-x_{j}(t)\right|=0 .
$$

5. Limit set modifications (qualitative changes to the system, e.g. modifications of bifurcation types).

However, this classification is primarily applicable to quite simple systems, primarily single-element ones. As for the multiagent systems, synchronization-type goals are usually of the most interest, since they relate to pattern emergence and complexity reduction possibilities. Indeed, in case all agent states converge to a single synchronous stable manifold, the whole system can then be controlled as one bunch of equally behaving components, thus requiring a single control input.

Recently, in the related works [Proskurnikov and Granichin, 2018; Granichin and Uzhva, 2020; Granichin et al., 2020] it was noticed that many artificial (and natural) complex systems exhibit so-called cluster synchronization (also refered to as clusterization), according to which agents synchronize in groups: system components from one group synchronize, while the ones belonging to different groups do not. For example, cluster synchronization occurs in human brain activity, assuming a brain can be accurately represented by a non-linear coupled oscillators model [Sadilek and Thurner, 2014]. According to the research provided in these articles, cluster synchronization mainly emerges in systems with incomplete connectivity between agents and due to external disturbances, which may affect connectivity and agent states.

In the phenomenon of clusterization, multiple synchronous stable manifolds form (or exist in our models), corresponding to separate clusters. We denote the number of such cluster manifolds $m$, and the following relation between the number of agents $N$ and number of clusters $m$ is often true:

$$
N \gg m \gg 1 .
$$

Equation (4) motivates the need to study clusterization phenomenon in complex systems for simple cluster control strategies development. In the current paper, we study the ways of cluster synchronization application to efficient system control strategy synthesis.

\section{Classification of Control Strategies}

In the previous Section, different types of goals for artificial complex system control were described. It was stated that both the specific type of a goal and the desired terminal system state values are regulated by the nature of the control input $u$. Current Section reveals possible approaches to the control input synthesis, regardless of the goal.

\subsection{Open-loop vs Feedback Control}

Amid the most simple yet straightforward ways to model and implement control action $u$ would be to construct a corresponding funcion $u_{i}(t)$ for each agent $i$, which only depends on time. For example, consider an ordinary linear system [Kalman, 1960]

$$
\dot{x}_{i}(t)=A_{i} x_{i}(t)+B_{i} u_{i}(t),
$$

where $A_{i}$ and $B_{i}$ are some matrices of an appropriate dimensionality, and $u_{i} \not \equiv 0$. By introducing such control function $u$, we obtain a nonhomogeneous system, the state $x_{i}$ of which changes independently on any functions of its current state, except the linear one. We further call such control approach a program control or openloop strategy, to emphasize its independence on the system state.

Frequently, in synchronization-type goals it is not enough for the control input $u$ to only depend on time, like in Equation (5). As an example, local voting [Amelina, 2013] and Kuramoto oscillator models [Acebron et al., 2005; Sadilek and Thurner, 2014; Benedetto et al., 2014; Chopra and Spong, 2006; Jadbabaie et al., 2005] demonstrate interesting complex behavior, provided corresponding control actions appear in relatively simple form due to the permission to use system state for control synthesis. Therefore, in contrast to the class of control inputs described above, we define a feedback (thus emphasizing its dependence on state) control strategy as a class of control functions $u_{i}(\boldsymbol{x}, t)$, which now also depend on a set of agent states $\boldsymbol{x}$. This set may contain the state vector $x_{i}$ and, for instance, state vectors of its neighbors (agents, which affect the agent $i$ ).

Real world systems are often subject to various disturbances, which may render agent states inaccurate for further feedback control. In [Proskurnikov and Granichin, 2018; Granichin and Uzhva, 2020], a concept of an observation was thoroughly discussed: basically, we assume that the precise agent state values may be unavailable, while we can only rely on a measurement procedure

$$
y_{j}\left(\boldsymbol{x}(t), v_{i}(t)\right)
$$

called an observation, with $\boldsymbol{x}(t)$ being a set of agent states (over which the observation is performed), and $v_{i}$ being the measurement error due to noise. Using this procedure, one can synthesize a control input $u_{i}(\boldsymbol{y}, t)$, where $\boldsymbol{y}$ is a set of all necessary observations of the corresponding agents.

Accordingly, we distinguish three classes of control strategies: 
1. Open-loop control.

2. State feedback control.

3. Observation feedback control.

\subsection{Optimal Control}

Another classification scheme of control strategies can be described in terms of the control action feasibility. Indeed, one is unable to apply infinite force to instantly achieve the desired system state; such force would always be limited by the abilities of the force actor: $|u(x, t)|<\infty$. The same reasoning is true for the system states $|x(t)|<\infty$ and observed outputs $|y(x, t)|<\infty$. Often, even stronger conditions on such functions may be regarded: for example, in [Galbraith and Vinter, 2003; Hernandez and Garcia, 2014] Lipschitz continuous control inputs are studied, where a function $x(t)$ is called Lipschitz continuous in case there exists a constant $K>0$ (so-called Lipschitz constant) such that

$$
\left|x\left(t_{1}\right)-x\left(t_{2}\right)\right| \leq K\left|t_{1}-t_{2}\right|
$$

is true for all real $t_{1}$ and $t_{2}$ (or for all $t_{1}$ and $t_{2}$ on the time interval under consideration). In other cases (e.g. in optimal control as optimization in Hilbert space), it might be required that $x, y$ or $u$ are bounded in $L_{p}(0, T)$ sense:

$$
\int_{0}^{T}|u(t)|^{p} d t<\infty
$$

for $u(t)$ as an example, where $T>0$ is terminal time (finite or infinite), up to which the system operates.

Recently, stability analysis and optimization techniques for control synthesis are of the most interest. The former seeks for such classes of control $u$, which lead to stable system states (or for ways to check if a given input $u$ leads to stable system states) [Lyapunov, 1892; Jadbabaie et al., 2005]. At the same time, the objective of the latter study is to find such $u$, which would lead the system to a stable manifold at the fastest rate [Kalman, 1960; Doyle, 1996; Le and Mendes, 2008]. Despite these two branches of complex system control study were initially clearly separated, nowadays they are closely related to each other. More than that, they became significantly more popular recently due to enough computational power available for numerical optimization methods, which are the only feasible solution to optimization for systems of significant complexity, intractable by analytical approaches.

In the situation of limited resourses, we can generally minimize one of the following values $J(u)$ for optimal control strategy synthesis.

1. Time consumption for convergence to the stable manifold, provided the control input is bounded:

$$
J_{1}(u)=\underset{u}{\arg \min } T(x, u),
$$

where $T$ is total time consumption as a fution of systems state $x$ and bounded control input $u$.

2. Conversely, control input, expressing "expended efforts", given the time is fixed and limited:

$$
J_{2}(u)=\underset{u}{\arg \min } \int_{0}^{T} \phi(x(t), u(x, t), t) d t,
$$

where $\phi$ is some function of systems state $x$, control input $u$ and time $t$.

We thus obtain two classes of control strategies, classified by optimality, further refered to as Equations (7) and (8). As an example, recall the system (5). In [Kalman, 1960] (for the number of agents $N=1$ ), control action $u(x, t)=-K x(t)$ called static-state feedback was shown to be optimal according to Equation (8).

In presence of noise, modifications to the optimization functionals become needed. In [Granichin and Fomin, 1986; Jerray et al., 2021], "minimax" control strategies are discussed, which first maximize the functional value with respect to the unknown or noise parameters, prior to minimization over the control inputs. In other words, only worst-case scenarios are considered.

It is worth noting that in many complex system control problems it is not possible to obtain an optimal control strategy analytically. In these cases, iterative gradient methods [Kelley, 1960; Polyak, 1964; Liang et al., 2020] allow to find an optimal solution in the following form:

$$
u_{k+1}=u_{k}-\gamma_{k} \nabla_{k} J\left(u_{k}\right),
$$

where $\gamma_{k}$ regulates the gradient descent speed.

\subsection{Control on Different Scales}

We further consider only synchronization-inducing and tracking control actions, as we deal with largescale multiagent complex systems. Due to the numerous amount of possible computation steps required to synthesize the desired optimal control strategies, we again return to the basic concept of information and signal compression. In [Proskurnikov and Granichin, 2018; Granichin and Uzhva, 2020; Granichin et al., 2020], first attempts to generalize the theory of complex multiagent system control, which utilizes clusterization, were proposed. It was shown how clusterization could reduce the number of required control inputs by the relation between the number of agents $N$ and clusters $m \ll N$. According to the results proposed in these papers, we distinguish three scale classes:

1. Local (microscopic) control, different for each agent.

2. Cluster (mesoscopic) control, different for all separate clusters.

3. Global (macroscopic) control, equal for all agents. 


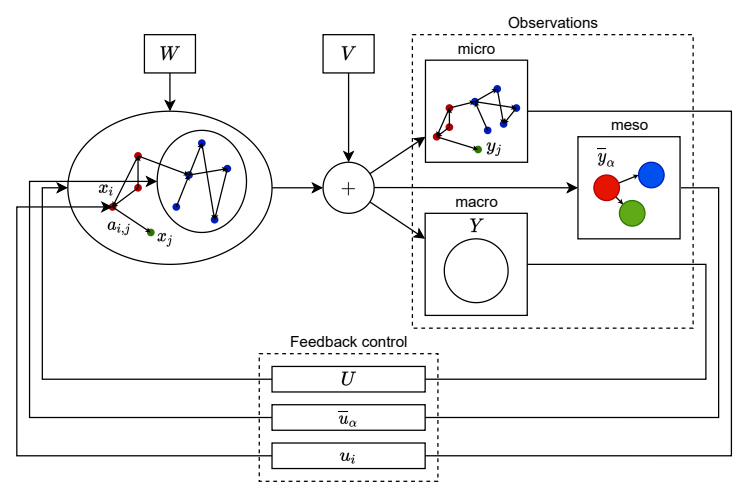

Figure 1. Observation feedback control for a multiagent complex system with clusterization. A system is represented as a composition of interacting agents, defined by their states $x_{i}$ and connected according to an adjacency matrix $A$ with elements $a_{i, j}$ equal to 1 in case a connection between agents $i$ and $j$ exists, otherwise equal to 0 . The system is affected by external disturbances $W$, which may change connectivity or agent states directly. Observations are exposed to noise $V$ and are divided into three levels: micro- (individual agents), macro(clusters) and mesoscopic (whole system). Provided the observations, control is separated into three types of inputs: $u_{i}$ ls local control input of an agent $i, \bar{u}_{\alpha}$ is a cluster control of a cluster $\alpha$, and $U$ is global control action equal for all agents.

We summarize possible scale classes by a scheme of the corresponding observation feedback control model with agent clusterization, see Figure 1.

Cluster control is possible for some integral (aggregated) cluster characteristics as separate cluster states, where clusters are assumed as separate subsystems. These characteristics can be obtained using, for example,

1. Local voting [Amelina, 2013].

2. "External" (i.e. non-multiagent) cluster identification methods, such as hierarchical clustering [Giordani et al., 2020; Karna and Gibert, 2021] or centroid-based clustering [Mughnyanti et al., 2020; Singh, 2021].

3. Data compression techniques, e.g. compressed sensing [Candès et al., 2006; Granichin et al., 2020].

\section{Optimal Cluster Control}

Consider a system of $N$ particles in an $K$-dimensional space, each of them having state $x_{i}[t]$, which is a vector in $\mathbb{R}^{K}$. Let the interactions between them led to clusterization $\mathcal{M}$ by the time $t=0$ : for example, due to the local voting. As it often happens in the real systems, the process of cluster formation is significantly faster than the cluster control processes. In other words, while the former usually occurs on a micro-level of fast simple inter-agent interactions, the latter requires certain substantial time for the observations of clusters to form and the corresponding control actions to be prepared. Thus, we further propose a discrete dynamical system model, which adequately resembles a computational control process with discrete time steps between the "observation" and "control" stages:

$$
x_{i}[t]=x_{i}[t-1]+c_{i}[t-1]+\frac{1}{\mu} f_{i}[t-1],
$$

where $c_{i}$ is the vector of "speed"; $f_{i}$ is the vector of "force" and $\mu$ is the "particle mass", equal for all particles.

Given the initial conditions for (9) are such that $\left|x_{i}[0]\right|>0$ and $\left|c_{i}[0]\right|>0$ (further in this Section, $|\cdot|$ norm is an arbitrary one, e.g. $\ell_{1}$ or $\ell_{2}$ ), our task is to bring all particles to a single point (let it be 0 , up to a change of variables) in one step, i.e. to minimize the following quality functional:

$$
J\left(f_{1}, \ldots, f_{N}\right)=\sum_{i=1}^{N}\left|x_{i}[1]\right|,
$$

with as little effort as possible. Below, we propose two control strategies: based on global- and cluster-state observations, then compare them. We define clusterization as in [Proskurnikov and Granichin, 2018; Granichin et al., 2020], for some $\varepsilon \geq\left|z_{i}-z_{j}\right|$ distance between agent cluster-observations in one cluster, and for $\delta \leq\left|z_{i}-z_{j}\right|$ distance between agent cluster-observations in different clusters. Further, we assume $z_{i}=x_{i}+c_{i}$.

\subsection{Global Control Strategy}

In global control, a common force is applied: $f_{i}=$ $F \forall i$. This force is applied to all particles, so that it effectively acts on the center of mass

$$
X[t]=\frac{1}{N} \sum_{i=1}^{N} x_{i}[t] .
$$

Accordingly, we define average particle speed as

$$
C[t]=\frac{1}{N} \sum_{i=1}^{N} c_{i}[t] .
$$

Thus $Z[t]=X[t]+C[t]$ plays the role of the observed global state and is used in deriving the optimal control strategy.

We denote $U:=F / \mu$ a two-component control input with the control value $U_{r}=|U|$ and the direction of control matching the direction of the force. After substituting (9) into (10) with the global controlling force, the quality functional

$$
\begin{aligned}
J_{\text {glob }}(U) & =\sum_{i=1}^{N}\left|x_{i}[0]+c_{i}[0]+U[0]\right|= \\
& =\sum_{i=1}^{N}\left|z_{i}[0]+U[0]\right|
\end{aligned}
$$


can be obtained. According to [Granichin and Fomin, 1986], an optimal solution to (13) is

$$
U_{\mathrm{opt}}[0]=-Z[0]=-(X[0]+C[0]),
$$

thus the minimum of (13) is

$$
\min J_{\text {glob }}(U)=\sum_{i=1}^{N}\left|z_{i}[0]-Z[0]\right|
$$

\subsection{Cluster Control Strategy}

Now, a common (for one cluster) force is applied to each agent: $f_{i}=\bar{f}_{\alpha} i \in \mathcal{M}_{\alpha}$, where $\mathcal{M}_{\alpha}$ is the collection of agent indices in cluster $\alpha$. In this case, such force acts on centers of mass of each cluster and average cluster particle speed correspondingly:

$$
\bar{x}_{\alpha}[t]=\frac{1}{M_{\alpha}} \sum_{i \in \mathcal{M}_{\alpha}} x_{i}[t], \bar{c}_{\alpha}[t]=\frac{1}{M_{\alpha}} \sum_{i \in \mathcal{M}_{\alpha}} c_{i}[t],
$$

where $M_{\alpha}=\left|\mathcal{M}_{\alpha}\right|$. Thus $\bar{z}_{\alpha}[t]=\bar{x}_{\alpha}[t]+\bar{c}_{\alpha}[t]$ plays the role of the observed cluster state and is used in deriving the optimal cluster control strategy.

We denote $\bar{u}_{\alpha}:=\bar{f}_{\alpha} / \mu$ a two-component control input with the control value $\bar{u}_{\alpha . r}=\left|\bar{u}_{\alpha}\right|$ and the direction of control matching the direction of the force for the cluster. The same reasoning as in the previous Subsection allows to obtain an optimal cluster control strategy

$$
\begin{aligned}
& \min J_{\text {clust }}\left(\bar{u}_{1}, \ldots, \bar{u}_{m}\right)= \\
= & \sum_{\alpha=1}^{m} \sum_{i \in \mathcal{M}_{\alpha}}\left|z_{i}[0]-\bar{z}_{\alpha}[0]\right|,
\end{aligned}
$$

where $m$ is the number of clusters and

$$
\bar{u}_{\alpha . \text { opt }}[0]=-\bar{z}_{\alpha}[0] .
$$

\subsection{Comparison of Cluster and Global Strategies}

Finally, we compare the two strategies in terms of their corresponding quality functionals (14) and (15) at optimal sets of parameters. It may be obvious that that the cluster control strategy is specifically "tuned" to change states of separated agent groups, while the global strategy roughly adjusts to the global state, which may vary significantly in different parts of the system-thus the latter is unable to reach that level of precision, achievable by the former. In fact, Equations (14) and (15) differ only in their corresponding control functions: $-Z[0]$ can be considered as a perturbed variant of $-\bar{z}_{\alpha}[0]$. Such perturbation takes the cluster control functional (15) out of its optimal value.

Summarizing the above, we propose a theorem, which anchors the mentioned conditions for the dynamical system (9) and the difference between the quality functionals (14) and (15).
Theorem 1. Consider the model (9) with $N$ agents, $x_{i}[0]>0$ and $c_{i}[0]>0$ for all $i$. Let the clusterization emerge by the time $t=0$, the number of clusters be equal to $m$ and each cluster have $M_{\alpha}$ agents, so that $\sum_{\alpha=1}^{m} M_{\alpha}=N$. Provided the optimized quality functionals (14) and (15) at their corresponding minima, it is true that if

$$
\left|\sum_{\alpha=1}^{m} M_{\alpha}\left(\bar{z}_{\alpha}[0]-Z[0]\right)\right|>0
$$

then

$$
\min J_{\text {clust }}<\min J_{\text {glob }}
$$

Proof. Theorem states that

$$
\sum_{\alpha=1}^{m} \sum_{i \in \mathcal{M}_{\alpha}}\left|z_{i}[0]-\bar{z}_{\alpha}[0]\right|<\sum_{i=1}^{N}\left|z_{i}[0]-Z[0]\right|
$$

under the condition (16). Consider a perturbed version of (15) with a non-zero perturbation $\gamma_{\alpha}$ :

$$
\sum_{\alpha=1}^{m} \sum_{i \in \mathcal{M}_{\alpha}}\left|z_{i}[0]-\bar{z}_{\alpha}[0]+\gamma_{\alpha}\right|,
$$

and let $\gamma_{\alpha}=\bar{z}_{\alpha}[0]-Z[0]$. It follows that

$$
\begin{gathered}
\sum_{\alpha=1}^{m} \sum_{i \in \mathcal{M}_{\alpha}}\left|z_{i}[0]-\bar{z}_{\alpha}[0]+\gamma_{\alpha}\right|- \\
-\sum_{\alpha=1}^{m} \sum_{i \in \mathcal{M}_{\alpha}}\left|z_{i}[0]-\bar{z}_{\alpha}[0]\right| \geq \\
\geq \mid N Z[0]-\sum_{\alpha=1}^{m} M_{\alpha} \bar{z}_{\alpha}[0]+\sum_{\alpha=1}^{m} M_{\alpha} \gamma_{\alpha}- \\
-N Z[0]+\sum_{\alpha=1}^{m} M_{\alpha} \bar{z}_{\alpha}[0]|=| \sum_{\alpha=1}^{m} M_{\alpha} \gamma_{\alpha} \mid>0
\end{gathered}
$$

Therefore

$$
\sum_{\alpha=1}^{m} \sum_{i \in \mathcal{M}_{\alpha}}\left(\left|z_{i}[0]-\bar{z}_{\alpha}[0]+\gamma_{\alpha}\right|-\left|z_{i}[0]-\bar{z}_{\alpha}[0]\right|\right)>0,
$$

and thus (18) is true for the chosen perturbation, which concludes the proof.

\subsection{Local Control Problem}

It was shown that the cluster control strategy allows for more precise agent state adjustment, than the global one. However, the most precise method is the local control, which targets each agent separately. If we consider the system (9), for which an optimal local control strategy $u_{i . \mathrm{opt}}[0]=-z_{i}[0]$ is applied, then the quality functional (10) (which we denote $\min J_{\text {loc }}\left(u_{1}, \ldots, u_{N}\right)$ for 
the chosen set of control inputs) becomes equal to 0 , which is the best possible solution.

Despite the local control strategy provides the most optimal set of control inputs, it is not feasible in many applications to complex systems with large number of elementary units. Indeed, finding $u_{i . \text { opt }}[0]$ requires measuring all the micro-states $x_{i}[0]$ and $c_{i}[0]$, which is often impossible. For example, when measuring neural oscillations of a brain, aggregated oscillatory states are usually observed in different brain areas on the mesoscale-in other words, on the level of multiple synchronized neurons. At the same time, it is significantly harder to measure activity of a single neuron due to both sophisticated measurement equipment and the big data arising in the necessity to record and process each separate neuron among multiple billions.

\section{Simultaneous Robust Local and Cluster Control}

Aiming to further develop the cluster control theory, we attempt to propose an approach to optimal local (microscopic) control synthesis in the presence of unknown but bounded disturbances and the model parameters, which allows to form clusters in a linear discrete multiagent system. We also introduce an approach to add cluster (mesoscopic) control to such model, which is based on the aggregated cluster characteristics. In our example case, these characteristics are obtained (by one of the approaches described in Section 3) as average agent state values in a specified cluster, assuming we know which agent belongs to which cluster in advance.

The linear model is as follows, assuming we use state feedback control (i.e. observation $y_{i} \equiv x_{i}$ ), and $i \in$ $\{1, \ldots, N\}=\mathcal{N}$, where $N$ is the number of agents:

$$
x_{i}[t]=\theta x_{i}[t-1]+u_{i}[t-1]+\bar{u}_{\alpha}[t-1]+w_{i}[t],
$$

where $x_{i}[t]$ is the agent $i$ state at time $t \in\{1, \ldots, T\} ; \theta$ is the model parameter (which defines agent inertia relatively to the control actions), the values of which are unknown, but belong to a bounded interval $\Theta ; u_{i}$ is the local control from a bounded interval $B_{u} ; \bar{u}_{\alpha}$ is the cluster control of the cluster $\alpha$ from a bounded interval $B_{\bar{u}}$; and $w_{i}$ is the disturbance, the values of which are unknown, but belong to a bounded interval $B_{w}$.

We further describe two most possible control strategies, based on corresponding cost functions:

1. Local synchronization-local control action, which lead each agent in a cluster toward each other.

2. Local tracking - local control, which aims to adjust agent state toward an aggregated state as a leading function.

In both cases, aggregated cluster states are calculated as

$$
\bar{x}_{\alpha}[t]=\frac{1}{M_{\alpha}} \sum_{j \in \mathcal{M}_{\alpha}} x_{j}[t],
$$

where $\mathcal{M}_{\alpha}$ is the set of agent indices in the cluster $\alpha$; and $M_{\alpha}=\left|\mathcal{M}_{\alpha}\right|$ is the number of agents in this cluster.
These states are adjusted by the mentioned additional set of cluster control inputs. We consider the tracking cluster control strategy, for some leading function $p_{\alpha}[t]$.

\subsection{Local Synchronization and Cluster Tracking}

In this case, we aim to minimize the distances between agent states in one cluster (local synchronization), as well as distances between an aggregated cluster state and its corresponding leading function-for each cluster (cluster tracking). We can formulate the local synchronization distance as follows:

$$
D_{i . \mathrm{S}}[t]=\sum_{\alpha=1}^{m} \chi_{i \in \mathcal{M}_{\alpha}} \sum_{j \in \mathcal{M}_{\alpha}}\left|x_{i}[t]-x_{j}[t]\right|,
$$

where $m$ is the number of clusters; $\chi_{i \in \mathcal{M}_{\alpha}}$ is an indicator function, which is equal to 1 in case agent $i$ belongs to the cluster $\alpha$, and is 0 otherwise.

At the same time, cluster tracking distance is

$$
\bar{D}_{\alpha}[t]=\left|\bar{x}_{\alpha}[t]-p_{\alpha}[t]\right|
$$

where $\bar{x}_{\alpha}$ is as in Equation (20); and $p_{\alpha}$ is the leading function.

Thus, we propose a corresponding minimax quality functional for the system (19), local synchronization (see Equation (21)) and cluster tracking (see Equation (22)) distances, minimization of which allows to find optimal control inputs on each time step:

$$
\begin{gathered}
J_{\mathrm{ST}}(\mathfrak{U}, \overline{\mathfrak{U}})= \\
=\sup _{\theta \in \Theta} \sup _{w_{j} \in B, j \in \mathcal{N}} \sum_{t=1}^{T}\left[\sum_{i=1}^{N} D_{i . \mathrm{S}}[t]+\sum_{\alpha=1}^{m} \bar{D}_{\alpha}[t]\right]
\end{gathered}
$$

where

$$
\begin{aligned}
\mathfrak{U} & =\{\boldsymbol{u}[1], \ldots, \boldsymbol{u}[T]\}, \\
\overline{\mathfrak{U}} & =\{\overline{\boldsymbol{u}}[1], \ldots, \overline{\boldsymbol{u}}[T]\}, \\
\boldsymbol{u}[t] & =\left\{u_{1}[t], \ldots, u_{N}[t]\right\}, \\
\overline{\boldsymbol{u}}[t] & =\left\{\bar{u}_{1}[t], \ldots, \bar{u}_{m}[t]\right\} .
\end{aligned}
$$

Minimization of (23) with respect to the local and cluster control inputs allows to find optimal values of $\mathfrak{U}$ :

$$
\{\mathfrak{U}, \overline{\mathfrak{U}}\}_{\text {opt.ST }}=\underset{\mathfrak{U} \in B_{u}, \overline{\mathfrak{U}} \in B_{\bar{u}}}{\arg \min } J_{\mathrm{ST}}(\mathfrak{U}) .
$$

\subsection{Local and Cluster Tracking}

Now we propose an alternative approach, where the distances between agent states and the corresponding aggregated state in one cluster (local tracking) are minimized. Local tracking distance is thus as follows:

$$
D_{i . \mathrm{T}}[t]=\sum_{\alpha=1}^{m} \chi_{i \in \mathcal{M}_{\alpha}}\left|x_{i}[t]-\bar{x}_{\alpha}[t]\right| .
$$


A corresponding minimax quality functional for the system (19), local tracking (see Equation (24)) and cluster tracking (see Equation (22)) distances, minimization of which allows to find optimal control inputs on each time step:

$$
\begin{gathered}
J_{\mathrm{TT}}(\mathfrak{U}, \overline{\mathfrak{U}})= \\
=\sup _{\theta \in \Theta} \sup _{w_{j} \in B, j \in \mathcal{N}} \sum_{t=1}^{T}\left[\sum_{i=1}^{N} D_{i . \mathrm{T}}[t]+\sum_{\alpha=1}^{m} \bar{D}_{\alpha}[t]\right] .
\end{gathered}
$$

Accordingly, optimal values of $\mathfrak{U}$ can be found by minimizing (25)

$$
\{\mathfrak{U}, \overline{\mathfrak{U}}\}_{\text {opt.TT }}=\underset{\mathfrak{U} \in B_{u}, \overline{\mathfrak{U}} \in B_{\bar{u}}}{\arg \min } J_{\mathrm{TT}}(\mathfrak{U}) .
$$

\section{Conclusion}

We proposed an approach to optimal cluster control synthesis for complex systems. First, we defined such systems as multiagent ones, through the definition of information and a system in general. Then, we described the ways to model them as dynamical systems, which support cluster control strategies. As for the cluster control, we further discussed how and why clusterization emerges in complex systems: the two main reasons are agent connectivity and external disturbances, as they prevent agents from communication and thus state synchronization or tracking. Next, we classified different control strategies: open-loop ones were compared with feedback control actions; various conditions for optimal control synthesis were discussed, as well as control on different scales (local, cluster and global). Aiming to demonstrate how cluster control outperforms global one, a simple linear noiseless model (9) was proposed, for which optimal control strategies minimizing quality functionals (14) and (15) were derived and compared: it was shown that the cluster strategy provides lower value of the functional in comaprison to the global approach. Finally, optimization problems for simultaneous robust local and cluster control synthesis were formulated for a linear multiagent model (19) by two approaches (synchronization and tracking) to treat local control: quality functionals (23) and (25) were derived. Furthermore, we plan to analyze the obtained functionals for possible solutions to the corresponding optimization problems. Moreover, we aim to consider possible modifications to the models (9) and (19), e.g. study noisy observations and time delays.

\section{Acknowledgments}

Sections 1-5 (except Subsection 4.4) were supported by the IPME RAS by Russian Science Foundation (project no. 21-19-00516). Subsection 4.4 was supported by the St. Petersburg State University (project No. 73555239).

\section{References}

Acebron, J., Bonilla, L., Pérez-Vicente, C., Farran, F., and Spigler, R. (2005). The Kuramoto model: A simple paradigm for synchronization phenomena. Reviews of Modern Physics, 77.

Amelina, N. (2013). Local voting protocol for decentralized load balancing of network with switched topology and noise in measurements. Vestnik St. Petersburg University: Mathematics, $\mathbf{4 6}$.

Arnold, V. and Silverman, R. (1987). Ordinary Differential Equations. Mit Press.

Benedetto, D., Caglioti, E., and Montemagno, U. (2014). On the complete phase synchronization for the $\mathrm{Ku}$ ramoto model in the mean-field limit. Communications in Mathematical Sciences, $\mathbf{1 3}$.

Candès, E., Romberg, J., and Tao, T. (2006). Robust uncertainty principles : Exact signal frequency information. Information Theory, IEEE Transactions on, 52, pp. 489-509.

Chopra, N. and Spong, M. (2006). On synchronization of Kuramoto oscillators. vol. 2005, 01, pp. 3916-3922.

Doyle, J. (1996). Robust and optimal control. In Proceedings of 35th IEEE Conference on Decision and Control, vol. 2, pp. 1595-1598.

Fradkov, A. (2007). Cybernetical Physics. Springer.

Galbraith, G. and Vinter, R. (2003). Lipschitz continuity of optimal controls for state constrained problems. SIAM J. Control and Optimization, 42, pp. 1727-1744.

Gazi, V. and Fidan, B. (2007). Coordination and control of multi-agent dynamic systems: Models and approaches. vol. 4433, 07, pp. 71-102.

Giordani, P., Ferraro, M., and Martella, F. (2020). Hierarchical Clustering, pp. 9-73.

Granichin, O. and Fomin, V. (1986). Dynamic programming method in minimax control problem. Vestnik Leningradskogo Universiteta, Matematika, 19(1), pp. 26-30.

Granichin, O. and Uzhva, D. (2020). Invariance preserving control of clusters recognized in networks of $\mathrm{Ku}-$ ramoto oscillators. 11.

Granichin, O., Uzhva, D., and Volkovich, Z. (2020). Cluster flows and multiagent technology. Mathematics, $\mathbf{9}$, pp. 22.

Hernandez, S. and Garcia, R. (2014). An observer for switched lipschitz continuous systems. International Journal of Control, 87.

Hu, S., Leung, C., and Leung, H. (2019). Modelling the dynamics of multiagent q-learning in repeated symmetric games: a mean field theoretic approach. In Advances in Neural Information Processing Systems, vol. 32, Curran Associates, Inc.

Jadbabaie, A., Motee, N., and Barahona, M. (2005). On the stability of the Kuramoto model of coupled nonlinear oscillators. vol. 5, 05, pp. 4296-4301.

Jerray, J., Fribourg, L., and André, E. (2021). An approximation of minimax control using random sampling and symbolic computation. IFAC-PapersOnLine, 54, pp. 265-270. 
Kalman, R. (1960). A new approach to linear filtering and prediction problems. Transactions of the ASMEJournal of Basic Engineering, 82 (Series D), pp. 3545.

Karna, A. and Gibert, K. (2021). Automatic identification of the number of clusters in hierarchical clustering. Neural Computing and Applications.

Kelley, H. (1960). Gradient theory of optimal flight paths. ARS Journal, 30, pp. 947-954.

Le, H. and Mendes, E. (2008). Optimal control of first order linear systems with fixed proportional-integral structure controller. In 2008 10th International Conference on Control, Automation, Robotics and Vision, pp. 1799-1804.

Li, H., Li, Y., and Zhao, H. V. (2020). Modeling decision process in multi-agent systems: A graphical markov game based approach. In 2020 Asia-Pacific Signal and Information Processing Association Annual Summit and Conference (APSIPA ASC), pp. 197-204.

Liang, D., Ma, F., and Li, W. (2020). New gradientweighted adaptive gradient methods with dynamic constraints. IEEE Access, 8, pp. 110929-110942.

Luo, G., Zhang, H., He, H., Li, J., and Wang, F. (2021). Multiagent adversarial collaborative learning via mean-field theory. IEEE Transactions on Cybernetics, 51 (10), pp. 4994-5007.

Lyapunov, A. (1892). The General Problem of the Stability of Motion. PhD thesis.

Mughnyanti, M., Efendi, S., and Zarlis, M. (2020). Anal- ysis of determining centroid clustering x-means algorithm with davies-bouldin index evaluation. IOP Conference Series: Materials Science and Engineering, 725.

Polyak, B. (1964). Some methods of speeding up the convergence of iteration methods. USSR Computational Mathematics and Mathematical Physics, 4(5), pp. 1-17.

Proskurnikov, A. and Granichin, O. (2018). Evolution of clusters in large-scale dynamical networks. Cybernetics and Physics, 7 (3), pp. 102-129.

Ravazzi, C., Dabbene, F., Lagoa, C., and Proskurnikov, A. (2021). Learning hidden influences in large-scale dynamical social networks: A data-driven sparsitybased approach, in memory of roberto tempo. IEEE Control Systems Magazine, 41 (5), pp. 61-103.

Sadilek, M. and Thurner, S. (2014). Physiologically motivated multiplex Kuramoto model describes phase diagram of cortical activity. Scientific Reports, $\mathbf{5}$.

Silva, E. (2014). DG-ABC: An Integrated Multi-Agent and Cellular Automata Urban Growth Model, pp. 5792.

Singh, S. (2021). Study of k-means and enhanced kmeans clustering algorithm. International Journal of Research in Computer Science, Vol. 4 Issue 10, pp. 103-107.

Strogatz, S. H. (2000). Nonlinear Dynamics and Chaos: With Applications to Physics, Biology, Chemistry and Engineering. Westview Press. 